\title{
MENNICKE SYMBOLS, $K$-COHOMOLOGY AND A BASS-KUBOTA THEOREM
}

\author{
J. FASEL
}

Abstract. If $A$ is a smooth algebra of dimension $d \geq 3$ over a perfect field $k$ of characteristic different from 2, then we show that the universal Mennicke symbol $M S_{d+1}(A)$ is isomorphic to the $K$-cohomology group $H^{d}\left(A, K_{d+1}\right)$. We then prove an analogue of the Bass-Kubota theorem for smooth affine surfaces over the algebraic closure of a finite field.

\section{INTRODUCTION}

As its title indicates, this paper mainly deals with Mennicke symbols. These symbols were extensively studied, for instance, in the solution of the congruence subgroup problem ([5]), the relations between Milnor $K$-theory and Quillen $K$ theory ([33]) and the computation of orbit sets of unimodular rows ([35]). One of the goals of this article is to show that if $A$ is a smooth algebra of dimension $d \geq 3$ over a perfect field $k$ of characteristic different from 2 , then the universal Mennicke symbol $M S_{d+1}(A)$ has a cohomological interpretation. More precisely, it is isomorphic to the $K$-cohomology group $H^{d}\left(A, K_{d+1}\right)$ (here it doesn't matter if we consider Milnor or Quillen $K$-groups). In this sense, this paper is a sequel of [16], where it was shown that the universal weak Mennicke symbol has a cohomological interpretation in the situation above. Weak Mennicke symbols were introduced by W. van der Kallen in order to understand orbit sets of unimodular rows, and it is clear by construction that there is a surjective homomorphism from the universal weak Mennicke symbol to the universal Mennicke symbol. Our cohomological approach allows us to compare these two symbols and derive some results on prestabilization in $K_{1}$. We are also able to compute the universal Mennicke symbol in some situations, for instance in the case of smooth rational real algebras, as well as to show that this symbol can be big over fields of small cohomological dimension. The methods we use can be seen as an extension of the methods used in [17] to prove that stably free modules of rank $d-1$ are free over smooth affine algebras of dimension $d$ over an algebraically closed field.

In the second part of the paper, we also study the Mennicke symbols of length 2. We use Grothendieck-Witt groups to prove that if $S$ is a smooth surface over an algebraically closed field $k$, then $S L_{2}(S) \cap E S p_{4}(S)=S L_{2}(S) \cap E_{3}(S)$. This allows us to deduce that for a smooth surface $S$ over the algebraic closure of a finite field,

$$
M S_{2}(S)=U m_{2}(S) / S L_{2}(S) \cap E_{3}(S)=U m_{2}(S) / S L_{2}(S) \cap E S p_{4}(S)=M S p_{2}(S),
$$

Received by the editors October 17, 2011 and, in revised form, November 7, 2012.

2010 Mathematics Subject Classification. Primary 13C10, 14C25, 14C35, 19A13, 19B14, 19E20; Secondary 19G38.

The author was partially supported by the Swiss National Science Foundation, grant PAOOP2_129089. 
which is an analogue of the Bass-Kubota theorem for Dedekind rings (see 20] for instance).

The organization of the paper is as follows. In Section 1, we first recall the definitions of a Mennicke symbol and of a weak Mennicke symbol of length $n \geq 3$ associated to a unimodular row of length $n \geq 3$. We then recall the definitions of the sheaves we will need for the comparison theorem. In particular, we introduce the $K$-cohomology groups $H^{i}\left(X, K_{j}\right)$ associated to a regular scheme $X$. We then state and prove the comparison theorem, which says that if $A$ is a smooth algebra of dimension $d \geq 3$ over a perfect field $k$ with $\operatorname{char}(k) \neq 2$, then the universal Mennicke symbol of length $d+1$ is isomorphic to $H^{d}\left(A, K_{d+1}\right)$. The proof is an easy consequence of the description of the universal weak Mennicke symbol obtained in [16, Theorem 4.9].

In Section 2, we use cohomological methods to compare the universal weak Mennicke symbol of length $d+1$ with the universal Mennicke symbol of length $d+1$ over a smooth algebra of dimension $d$. We also obtain divisibility results for universal Mennicke symbols. We then perform the computation of $M S_{d+1}(A)$ when $A$ is a smooth rational real algebra of dimension $d$ before focusing on stabilization questions. We finally prove that over smooth algebras of odd dimension $d$ over a field, $M S_{d+1}(A)$ is sufficient to understand the stably free modules of rank $d$.

The proof that $S L_{2}(S) \cap E S p_{4}(S)=S L_{2}(S) \cap E_{3}(S)$ for a smooth surface $S$ over an algebraically closed field takes place in Section 3 . We start with a quick reminder of basic results on higher Grothendieck-Witt groups, including the definitions. These groups are used to prove that if $S$ is a smooth surface over an algebraically closed field of characteristic different from 2, then $K_{1} S p(S)$ injects into $S K_{1}(S)$, yielding the result. We then introduce stable Mennicke symbols of length 2 before proving the Bass-Kubota theorem.

Conventions. The fields considered are of characteristic different from 2 . If $X$ is a scheme over a field $k$ and $x_{p} \in X^{(p)}$, we denote by $\mathfrak{m}_{p}$ the maximal ideal in $\mathcal{O}_{X, x_{p}}$ and by $k\left(x_{p}\right)$ its residue field. Finally $\omega_{x_{p}}$ will denote the $k\left(x_{p}\right)$-vector space $\operatorname{Ext}_{\mathcal{O}_{X, x_{p}}}^{p}\left(k\left(x_{p}\right), \mathcal{O}_{X, x_{p}}\right)$ (which is one-dimensional if $X$ is regular at $x_{p}$ ).

\section{Symbols And COHOMOLOGY}

In this section, we recall the definitions of Mennicke symbols and weak Mennicke symbols. We then explore their links to the cohomology groups of some sheaves. The main result is the comparison theorem, which says that for a smooth affine algebra $A$ of dimension $d \geq 3$ over some perfect field $k$ (with $\operatorname{char}(k) \neq 2$ ), the universal Mennicke symbol of length $d$ is isomorphic to the $K$-cohomology group $H^{d}\left(A, K_{d+1}\right)$.

1.1. Unimodular rows. Let $R$ be a ring. A unimodular row of length $n \geq 2$ is a row $a=\left(a_{1}, \ldots, a_{n}\right)$ with $a_{i} \in R$ such that there exist $b_{1}, \ldots, b_{n} \in R$ with $\sum a_{i} b_{i}=1$. We denote by $\operatorname{Um}_{n}(R)$ the set of unimodular rows of length $n$ and consider it as a pointed set with base point $e_{1}:=(1,0, \ldots, 0)$. If $M \in G L_{n}(R)$ and $a \in U m_{n}(R)$, then $a M$ is also unimodular, and thus $U m_{n}(R)$ is endowed with a right action of $G L_{n}(R)$. Of course, any subgroup of $G L_{n}(R)$ acts, and one is classically interested in the (pointed) orbit set $U m_{n}(R) / S L_{n}(R)$, which classifies up to isomorphism projective modules such that $P \oplus R \simeq R^{n}$, and $U m_{n}(R) / E_{n}(R)$, which classifies unimodular rows up to elementary homotopies and is endowed with 
an abelian group structure when $n$ is reasonable compared to the Krull dimension $d$ of $R$ ([36, Theorem 4.1]). A way to understand unimodular rows is through symbols, as explained in the next two sections.

1.2. Mennicke symbols. A Mennicke symbol of length $n \geq 3$ is a pair $(\phi, G)$ where $G$ is a group and

$$
\phi: U m_{n}(R) \rightarrow G
$$

is a map such that the two following properties are satisfied:

ms1. $\phi(1,0, \ldots, 0)=1$ and $\phi(v)=\phi(w M)$ if $M \in E_{n}(R)$.

ms2. $\phi\left(a, a_{2}, \ldots, a_{n}\right) \cdot \phi\left(b, a_{2}, \ldots, a_{n}\right)=\phi\left(a b, a_{2}, \ldots, a_{n}\right)$ for any unimodular rows $\left(a, a_{2}, \ldots, a_{n}\right)$ and $\left(b, a_{2}, \ldots, a_{n}\right)$.

It is clear from the definition that a universal Mennicke symbol $\left(m s, M S_{n}(R)\right)$ exists.

1.3. Weak Mennicke symbols. A weak Mennicke symbol of length $n \geq 3$ is a pair $(\phi, G)$ where $G$ is a group and

$$
\phi: U m_{n}(R) \rightarrow G
$$

is a map such that the two following properties are satisfied:

wms1. $\phi(1,0, \ldots, 0)=1$ and $\phi(v)=\phi(w M)$ if $M \in E_{n}(R)$.

wms2. $\phi\left(a, a_{2}, \ldots, a_{n}\right) \cdot \phi\left(1-a, a_{2}, \ldots, a_{n}\right)=\phi\left(a(1-a), a_{2}, \ldots, a_{n}\right)$ for any unimodular row $\left(a, a_{2}, \ldots, a_{n}\right)$ such that $\left(1-a, a_{2}, \ldots, a_{n}\right)$ is also unimodular.

The definition of a weak Mennicke symbol, due to W. van der Kallen ([36, §1.3]), was originally more complicated. However, van der Kallen could show that in most of the situations the original definition is the same as the definition given above ([37, Theorem 3.3]).

Again, it is clear that a universal weak Mennicke symbol $\left(w m s, W M S_{n}(R)\right)$ exists. Since a Mennicke symbol of length $n$ is obviously a weak Mennicke symbol of length $n$, there is a unique homomorphism $F: W M S_{n}(R) \rightarrow M S_{n}(R)$ such that the diagram

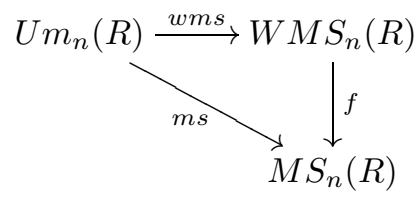

commutes. Observe that $f$ is surjective by definition.

1.4. Cohomology. Let $F$ be a field. For any $n \in \mathbb{N}$, we denote by $K_{n}(F)$ the $n$-th Milnor $K$-theory group of $F$. We also denote by $W(F)$ the Witt ring of $F$, and for any $n \in \mathbb{Z}$ by $I^{n}(F)$ the $n$-th power of the fundamental ideal in $W(F)$ (with the convention that $I^{n}(F)=W(F)$ when $\left.n \leq 0\right)$. If $L$ is an $F$-vector space of dimension 1 , then we can also consider the twisted Witt group $W(F, L)$ (classifying vector spaces $V$ endowed with symmetric anisotropic isomorphisms $\left.V \rightarrow \operatorname{Hom}_{F}(V, L)\right)$. Choosing a generator of $L$ yields an isomorphism $W(F) \rightarrow W(F, L)$ under which we can consider the images of $I^{n}(F)$. The resulting subgroups $I^{n}(F, L) \subset W(F, L)$ are independent of the choice of the generator of $L$ ([14, Lemma E.1.2]).

By definition, $I^{n+1}(F, L) \subset I^{n}(F, L)$ for any $n$, and it turns out that the quotient is canonically isomorphic to $I^{n}(F) / I^{n+1}(F):=\bar{I}^{n}(F)$ ([14, Lemma E.1.3]). There 
is a homomorphism $s_{n}: K_{n}(F) \rightarrow \bar{I}^{n}(F)$ defined on symbols by ([23, Theorem 4.1])

$$
s_{n}\left(\left\{a_{1}, \ldots, a_{n}\right\}\right)=\left\langle-1, a_{1}\right\rangle \otimes \ldots \otimes\left\langle-1, a_{n}\right\rangle .
$$

Note that $s_{n}$ induces an isomorphism $K_{n}(F) / 2 K_{n}(F) \rightarrow \bar{I}^{n}(F)$ by [1] and 25. We define $G^{n}(F, L)$ following [4, $\left.\S 1\right]$ as the fibre product

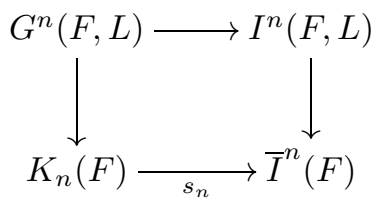

Observe that $G^{n}(F, L)$ coincides with the (twisted) Milnor-Witt $K$-group $K_{n}^{M W}(F, L)$ defined in [24, Definition 5.1] (see [24, Theorem 5.3]).

Let $X$ be a regular connected scheme over $k$. If $U \subset X$ is an open subset we can consider the kernel of the residue homomorphism ([23, Lemma 2.1]):

$$
K_{n}(k(U)) \stackrel{d_{K}}{\longrightarrow} \bigoplus_{x \in U^{(1)}} K_{n-1}(k(x)) .
$$

This defines a presheaf on $X$, whose associated sheaf we denote by $K_{n}$. For the properties of the cohomology groups of this sheaf, we refer the reader to [19] or [27.

Similarly, we can consider for any $U \subset X$ the kernel of the residue homomorphism ([14, Chapter 7] and [14, Lemma 9.2.3])

$$
I^{n}(k(U)) \stackrel{d_{I}}{\longrightarrow} \bigoplus_{x \in U^{(1)}} I^{n-1}\left(k(x), \omega_{x}\right) .
$$

We denote by $I^{n}$ the sheaf associated to this presheaf and by $\bar{I}^{n}$ the quotient $I^{n} / I^{n+1}$. Since $d_{K}$ and $d_{I}$ are compatible ([14, Theorem 10.2.6]), we also get a residue homomorphism on $G^{n}(k(U))$ and a sheaf $G^{n}$ on $X$. Observe that by definition there is an exact sequence of sheaves on $X$ :

$$
0 \longrightarrow I^{n+1} \longrightarrow G^{n} \longrightarrow K_{n} \longrightarrow 0 \text {. }
$$

For the properties of the sheaves $I^{n+1}$ and $G^{n}$, we refer the reader to [14, $\S 9.3$, $\S 10.4]$ and [13.

The homomorphisms $s_{n}: K_{n}(F) \rightarrow \bar{I}^{n}(F)$ induce a homomorphism of sheaves $K_{n} / 2 K_{n} \rightarrow \bar{I}^{n}$, which is an isomorphism (again by [41] and [25]). These two sheaves are yet isomorphic to a third one: For any $U \subset X$ and any prime number $l$, consider the kernel of the residue homomorphism

$$
H_{e t}^{n}\left(k(U), \mu_{l}^{\otimes n}\right) \longrightarrow \bigoplus_{x \in U^{(1)}} H_{e t}^{n-1}\left(k(x), \mu_{l}^{\otimes n-1}\right)
$$

defined for instance in [27]. We denote by $\mathcal{H}^{n}(l)$ the sheaf associated to this presheaf. There is a natural homomorphism $K_{n} \rightarrow \mathcal{H}^{n}(l)$ ([23, Lemma 6.1]), which yields a homomorphism $K_{n} / l \rightarrow \mathcal{H}^{n}(l)$. This is an isomorphism by Voevodsky's work [41] and [25] when $l=2$, by Merkurjev and Suslin when $n=2$ ([21]), and by Voevodsky-Suslin-Rost with Weibel's patch when $l \neq 2$ and $n \geq 3$ ([42]). One of the features of the sheaf $\mathcal{H}^{n}(l)$ is the Bloch-Ogus spectral sequence ([9]). This spectral sequence is defined at page 2 by $E_{2}^{p, q}:=H_{Z a r}^{p}\left(X, \mathcal{H}^{q}(l)\right)$ and converges to $H_{e t}^{p+q}\left(X, \mu_{l}\right)$. 
1.5. Comparison theorems. Let $A$ be a smooth algebra of dimension $d$ over a field $k$. Recall from [16. Theorem 2.1] that $U m_{d+1}(A) / E_{d+1}(A)$ can be identified with morphisms of schemes $\operatorname{Spec}(A) \rightarrow \mathbb{A}^{d+1}-\{0\}$ up to naive homotopies. A straightforward computation shows that $H^{d}\left(\mathbb{A}^{d+1}-\{0\}, G^{d+1}\right)=G W(k)$, where the latter is the Grothendieck-Witt group of $k([16, \S 3.3])$. We obtain in this way a map

$$
\phi: U m_{d+1}(A) / E_{d+1}(A) \rightarrow H^{d}\left(A, G^{d+1}\right)
$$

defined by $v \mapsto v^{*}(\langle 1\rangle)$, where $v: \operatorname{Spec}(A) \rightarrow \mathbb{A}^{d+1}-\{0\}$ is a unimodular row and $v^{*}: H^{d}\left(\mathbb{A}^{d+1}-\{0\}, G^{d+1}\right) \rightarrow H^{d}\left(A, G^{d+1}\right)$ is the pull-back homomorphism defined in [13, Definition 7.1]. It turns out that $\phi$ is a weak Mennicke symbol and therefore induces a homomorphism ([16, Theorem 4.1])

$$
\Phi: W M S_{d+1}(A) \rightarrow H^{d}\left(A, G^{d+1}\right) .
$$

Theorem 1.1. Let $A$ be a smooth algebra of dimension $d \geq 3$ over a perfect field $k$ with $\operatorname{char}(k) \neq 2$. Then the homomorphism

$$
\Phi: W M S_{d+1}(A) \rightarrow H^{d}\left(A, G^{d+1}\right)
$$

is an isomorphism.

Proof. See [16, Theorem 4.9].

Remark 1.2. In [16], we could only prove that $W M S_{d+1}(A) \rightarrow H^{d}\left(A, G^{d+1}\right)$ was an isomorphism for $d \geq 3$. However, this result is also true for $d=2$, as we will show in further work.

We will now prove that the universal Mennicke symbol $M S_{d+1}(A)$ is isomorphic to $H^{d}\left(A, K_{d+1}\right)$ under the same hypotheses as in Theorem 1.1. We first define a map

$$
\psi: U m_{d+1}(A) / E_{d+1}(A) \rightarrow H^{d}\left(A, K_{d+1}\right)
$$

as the composition

$$
U m_{d+1}(A) / E_{d+1}(A) \stackrel{\phi}{\longrightarrow} H^{d}\left(A, G^{d+1}\right) \longrightarrow H^{d}\left(A, K_{d+1}\right),
$$

where the right homomorphism is the homomorphism induced by the map of sheaves $G^{d+1} \rightarrow K_{d+1}$.

Lemma 1.3. The map $\psi$ induces a homomorphism

$$
\Psi: M S_{d+1}(A) \rightarrow H^{d}\left(A, K_{d+1}\right) .
$$

Proof. It suffices to prove that $\psi$ satisfies relation ms2. We can follow 16, proof of Theorem 4.1] with $K_{d+1}$ instead of $G^{d+1}$ to get the result.

Theorem 1.4. Let $A$ be a smooth algebra of dimension $d \geq 3$ over a perfect field $k$ of characteristic different from 2. Then $\Psi: M S_{d+1}(R) \rightarrow H^{d}\left(A, K_{d+1}\right)$ is an isomorphism.

Proof. Observe first that the following diagram is commutative:

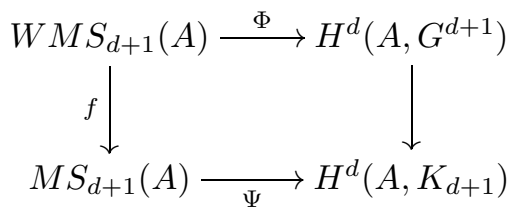


Indeed, $H^{d}\left(A, K_{d+1}\right)$ being a Mennicke symbol, it is also a weak Mennicke symbol. There is then a unique homomorphism $W M S_{d+1}(A) \rightarrow H^{d}\left(A, K_{d+1}\right)$ extending $\psi$. Since both compositions in the diagram do the job, they are equal.

Consider now the following commutative diagram:

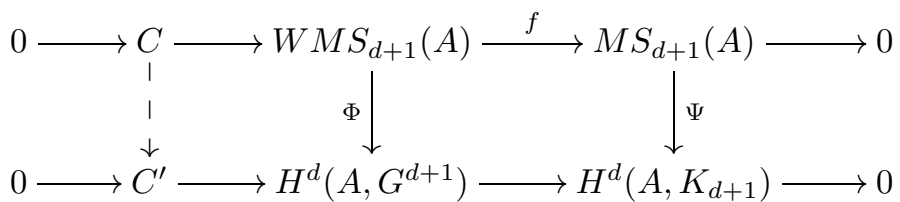

where $C$ and $C^{\prime}$ are the kernels of the horizontal homomorphisms. Since $\Phi$ is an isomorphism by Theorem 1.1, it suffices to prove that the induced homomorphism $C \rightarrow C^{\prime}$ is surjective to conclude. Using the exact sequence of sheaves

$$
0 \longrightarrow I^{d+2} \longrightarrow G^{d+1} \longrightarrow K_{d+1}^{M} \longrightarrow 0
$$

we get a surjective homomorphism $H^{d}\left(A, I^{d+2}\right) \rightarrow C^{\prime}$.

Let $\mathfrak{m}$ be a maximal ideal of $A$. Arguing as in [7, Corollary 2.4], we see that there exists a regular sequence $\left(v_{1}, \ldots, v_{d}\right) \subset A$ such that

$$
A /\left(v_{1}, \ldots, v_{d}\right)=A / \mathfrak{m} \times A / M_{1} \times \ldots \times A / M_{r},
$$

where the $M_{i}$ are $\mathfrak{m}_{i}$-primary ideals for some distinct maximal ideals $\mathfrak{m}_{1}, \ldots, \mathfrak{m}_{r}$ (also distinct of $\mathfrak{m}$ ). Let

$$
\psi_{v_{1}, \ldots, v_{d}}: A /\left(v_{1}, \ldots, v_{d}\right) \rightarrow \operatorname{Ext}_{A}^{d}\left(A /\left(v_{1}, \ldots, v_{d}\right), A\right)
$$

be the isomorphism defined by $\psi_{v_{1}, \ldots, v_{d}}(1)=\operatorname{Kos}\left(v_{1}, \ldots, v_{d}\right)$, where the latter is the Koszul complex associated to the regular sequence $\left(v_{1}, \ldots, v_{d}\right)$. Using the same argument as in [16, $\S 4.1]$, we see that $I^{2}\left(A / \mathfrak{m}, \omega_{\mathfrak{m}}\right)$ is generated by elements of the form $\langle-1, a\rangle \otimes\langle-1, b\rangle \cdot \psi_{v_{1}, \ldots, v_{d}}$ with $\left(a, v_{1}, \ldots, v_{d}\right)$ and $\left(b, v_{1}, \ldots, v_{d}\right)$ unimodular. Doing the same for all maximal ideals $\mathfrak{m} \subset A$, we get a set of generators of $H^{d}\left(A, I^{d+2}\right)$. The image in $C^{\prime}$ of such a generator is of the form

$$
\left(a b,\langle-1, a b\rangle \cdot \psi_{v_{1}, \ldots, v_{d}}\right)-\left(a,\langle-1, a\rangle \cdot \psi_{v_{1}, \ldots, v_{d}}\right)-\left(b,\langle-1, b\rangle \cdot \psi_{v_{1}, \ldots, v_{d}}\right) .
$$

This is precisely the image of

$$
w m s\left(a b, v_{1}, \ldots, v_{d}\right)-w m s\left(a, v_{1}, \ldots, v_{d}\right)-w m s\left(b, v_{1}, \ldots, v_{d}\right)
$$

under the homomorphism $\Phi: W M S_{d+1}(A) \rightarrow H^{d}\left(A, G^{d+1}\right)$. Now by definition the element $w m s\left(a b, v_{1}, \ldots, v_{d}\right)-w m s\left(a, v_{1}, \ldots, v_{d}\right)-w m s\left(b, v_{1}, \ldots, v_{d}\right)$ vanishes in $M S_{d+1}(A)$, whence the result.

This cohomological description allows us to perform some computations of the universal Mennicke symbol of length $d+1$, as we will see in the next section.

\section{Prestabilization and stably free modules}

2.1. Preliminary computations. As in the previous section, $A$ is a smooth algebra of dimension $d \geq 3$ over a perfect field $k$ with $\operatorname{char}(k) \neq 2$.

Theorem 2.1. Let $k$ be a perfect field such that c.d.2 $(k) \leq 2$ and let $A$ be a smooth affine algebra of dimension $d \geq 3$ over $k$. Then the homomorphism

$$
W M S_{d+1}(A) \rightarrow M S_{d+1}(A)
$$

is an isomorphism. 
Proof. In view of Theorem 1.4, it suffices to show that the homomorphism

$$
H^{d}\left(A, G^{d+1}\right) \rightarrow H^{d}\left(A, K_{d+1}\right)
$$

is an isomorphism. Using the exact sequence of sheaves

$$
0 \longrightarrow I^{d+2} \longrightarrow G^{d+1} \longrightarrow K_{d+1}^{M} \longrightarrow 0,
$$

we see that it suffices to prove that $H^{d}\left(A, I^{d+2}\right)=0$. Observe that the group $H^{d}\left(A, I^{d+2}\right)$ can be computed by using a flasque resolution of $I^{d+2}$, the (filtered) Gersten-Witt complex of $A$ ([13, Theorem 3.11]):

$$
I^{d+2}(k(X)) \rightarrow \ldots \rightarrow \bigoplus_{x_{d-1} \in X^{(d-1)}} I^{3}\left(k\left(x_{d-1}\right), \omega_{x_{d-1}}\right) \rightarrow \bigoplus_{x_{d} \in X^{(d)}} I^{2}\left(k\left(x_{d}\right), \omega_{x_{d}}\right),
$$

where $X=\operatorname{Spec}(A)$.

If $x_{i} \in X^{(i)}$, then $k\left(x_{i}\right)$ is of cohomological dimension $c . d_{.2}\left(k\left(x_{i}\right)\right) \leq d+2-i$ by [31, $\S 4.2$, Proposition 11]. It follows that $H_{e t}^{d+3-i}\left(k\left(x_{i}\right), \mu_{2}\right)=0$ and then $I^{d+3-i}\left(k\left(x_{i}\right)\right) / I^{d+4-i}\left(k\left(x_{i}\right)\right)=0$ by [25, Theorem 4.1] and [41, Theorem 7.4]. The Arason-Pfister Hauptsatz ([1]) then shows that $I^{d+3-i}\left(k\left(x_{i}\right)\right)=0$. Therefore $H^{i}\left(A, I^{d+2}\right)=H^{i}\left(A, \bar{I}^{d+2}\right)$ for any $i \in \mathbb{N}$ and $H^{i}\left(A, \bar{I}^{j}\right)=0$ for any $i \in \mathbb{N}$ and $j \geq d+3$. Now $H^{i}\left(A, \bar{I}^{j}\right) \simeq H^{i}\left(A, \mathcal{H}^{j}(2)\right.$ ) (as seen in Section 1.4), and inspection of the Bloch-Ogus spectral sequence shows that $H^{d}\left(A, \bar{I}^{d+2}\right) \simeq H_{e t}^{2 d+2}\left(A, \mu_{2}\right)$. The latter is trivial since $A$ is affine of dimension $d$ over a field of cohomological dimension at most 2 ([22, Chapter VI, Theorem 7.2] and [22, Chapter III, Theorem $2.20])$.

Following the arguments of [17, Proposition 6.1], we can also prove the following theorem.

Theorem 2.2. Let $A$ be a smooth algebra of dimension $d \geq 3$ over a perfect field $k$ with c.d. $(k) \leq 1$. Then $M S_{d+1}(A)$ is uniquely divisible prime to the characteristic of $k$.

Proof. Let $l$ be a prime number with $l \neq \operatorname{char}(k)$. We consider the exact sequences of sheaves (see [8, proof of Corollary 1.11] for instance) given by the multiplication by $l$ on sheaves

$$
0 \longrightarrow K_{l+1} \longrightarrow K_{d+1} \longrightarrow l K_{d+1} \longrightarrow 0
$$

and

$$
0 \longrightarrow l K_{d+1} \longrightarrow K_{d+1} \longrightarrow K_{d+1} / l \longrightarrow 0 .
$$

The second sequence yields a long exact sequence in cohomology which ends with

$$
H^{d-1}\left(A, K_{d+1} / l\right) \longrightarrow H^{d}\left(A, l K_{d+1}\right) \rightarrow H^{d}\left(A, K_{d+1}\right) \longrightarrow H^{d}\left(A, K_{d+1} / l\right) \longrightarrow 0 .
$$

Now the groups $H^{i}\left(A, K_{d+1} / l\right)$ are isomorphic to $H^{i}\left(A, \mathcal{H}^{d+1}(l)\right)$ (see Section 1.4). As in the above proof, $k\left(x_{i}\right)$ is of cohomological dimension c.d.2 $\left(k\left(x_{i}\right)\right) \leq d+$ $1-i$ by [31, $\S 4.2$, Proposition 11] when $x_{i} \in X^{(i)}$. Then inspecting the BlochOgus spectral sequence, we get an epimorphism $H_{e t}^{2 d}\left(A, \mu_{l}\right) \rightarrow H^{d-1}\left(A, K_{d+1} / l\right)$ and an isomorphism $H^{d}\left(A, K_{d+1} / l\right) \simeq H_{e t}^{2 d+1}\left(A, \mu_{l}\right)$. Since $A$ is affine, both étale cohomology groups are trivial by [22, Chapter VI, Theorem 7.2] and [22, Chapter III, Theorem 2.20]. It then follows that $H^{d}\left(A, l K_{d+1}\right) \simeq H^{d}\left(A, K_{d+1}\right)$. 
We next use the first exact sequence of sheaves. This yields

$$
H^{d-1}\left(A, l K_{d+1}\right) \longrightarrow H^{d}\left(A,{ }_{l} K_{d+1}\right) \longrightarrow H^{d}\left(A, K_{d+1}\right) \longrightarrow H^{d}\left(A, l K_{d+1}\right) \longrightarrow 0 .
$$

Using our result in the previous paragraph, this sequence becomes

$$
H^{d-1}\left(A, l K_{d+1}\right) \rightarrow H^{d}\left(A,{ }_{l} K_{d+1}\right) \rightarrow H^{d}\left(A, K_{d+1}\right) \stackrel{\cdot l}{\rightarrow} H^{d}\left(A, K_{d+1}\right) \longrightarrow 0 .
$$

It then suffices to prove that the left homomorphism is surjective to conclude. The second exact sequence of sheaves yields a homomorphism $H^{d-2}\left(A, K_{d+1} / l\right) \rightarrow$ $H^{d-1}\left(A, l K_{d+1}\right)$ and we consider the diagram

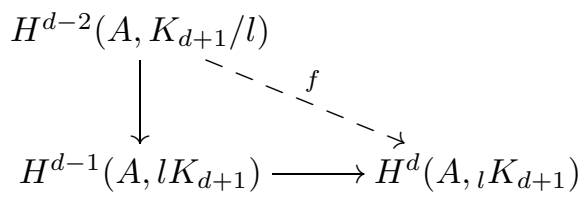

Suppose that $k$ contains a primitive $l$-th root of unity $\xi$. For any field $k \subset F$ and any $n \geq 1$, there is a homomorphism $K_{n-1}(F) / l \rightarrow{ }_{l} K_{n}(F)$ defined on symbols by $\left\{a_{1}, \ldots, a_{n-1}\right\} \mapsto\left\{\xi, a_{1}, \ldots, a_{n-1}\right\}$. It is not hard to see that this induces a morphism of sheaves $K_{d} / l \rightarrow{ }_{l} K_{d+1}$. It follows from [34, Theorem 1.8] that this morphism of sheaves induces an isomorphism $H^{d}\left(A, K_{d} / l\right) \rightarrow H^{d}\left(A,{ }_{l} K_{d+1}\right)$. If we denote by $E_{2}^{p q}$ the terms at page 2 in the Bloch-Ogus spectral sequence, the homomorphism $f$ then reads as

$$
f: E_{2}^{d-2, d+1} \rightarrow E_{2}^{d, d}
$$

and is precisely the differential at page 2 by [2, Proposition 7.5]. Now $E_{3}^{d, d}$ injects in $H_{e t}^{2 d}\left(A, \mu_{l}\right)$, and we have seen above that the right hand term is trivial. Therefore $f$ is surjective and

$$
\cdot l: H^{d}\left(A, K_{d+1}\right) \rightarrow H^{d}\left(A, K_{d+1}\right)
$$

is an isomorphism.

If $k$ doesn't contain a primitive $l$-th root of unity, it is enough to show that $f: H^{d-2}\left(A, K_{d+1} / l\right) \rightarrow H^{d}\left(A,{ }_{l} K_{d+1}\right)$ is still surjective to conclude. There exists a finite separable extension $k \subset L$ of degree prime to $l$ such that $L$ contains a primitive $l$-th root of unity. If we denote by $A_{L}$ the algebra $A \otimes_{k} L$, we see that the morphism $g: \operatorname{Spec}\left(A_{L}\right) \rightarrow \operatorname{Spec}(A)$ is finite and étale. A simple computation shows that the composition $g_{*} g^{*}: H^{d}\left(A,{ }_{l} K_{d+1}\right) \rightarrow H^{d}\left(A,{ }_{l} K_{d+1}\right)$ is the multiplication by $[L: k]$ and is therefore surjective. It follows that $g_{*}$ is surjective, and we can conclude from the commutative diagram

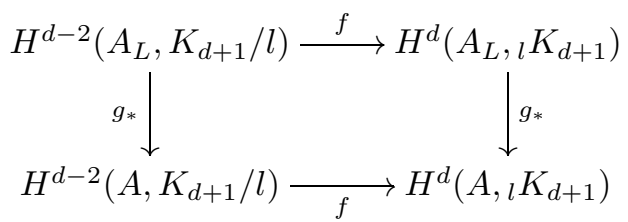

that the lower $f$ is surjective.

When the base field is of cohomological dimension greater than 2, then Theorem 2.1 is no longer true. As a simple illustration of this fact, we consider varieties over $\mathbb{R}$. Recall that a smooth connected variety $X$ of dimension $d$ over $\mathbb{R}$ is rational if $X \times \operatorname{Spec}(\mathbb{C})$ is birational to $\mathbb{P}_{\mathbb{C}}^{d}$. We begin with the computation of $M S_{d+1}$. 
Theorem 2.3. Let $A$ be a smooth rational $\mathbb{R}$-algebra of dimension $d \geq 3$. Then

$$
M S_{d+1}(A) \simeq H^{d}(X(\mathbb{R}), \mathbb{Z} / 2),
$$

where $X=\operatorname{Spec}(A)$ and the right hand term denotes the singular cohomology of the real manifold $X(\mathbb{R})$.

Proof. First recall from [16, Proposition 5.4] that there is an exact sequence

$$
H^{d}\left(X \times \operatorname{Spec}(\mathbb{C}), K_{d+1}\right) \stackrel{f_{*}}{\longrightarrow} H^{d}\left(X, K_{d+1}\right) \longrightarrow H^{d}\left(X, K_{d+1} / 2 K_{d+1}\right) \longrightarrow 0
$$

where $f_{*}$ is the push-forward associated to the (finite) morphism $X \times \operatorname{Spec}(\mathbb{C}) \rightarrow X$. Since $A$ is rational, $H^{d}\left(X \times \operatorname{Spec}(\mathbb{C}), K_{d+1}\right)=0$ by [16, Proposition 5.6]. Now $H^{d}\left(X, K_{d+1} / 2 K_{d+1}\right) \simeq H^{d}\left(X, \mathcal{H}^{d+1}(2)\right)$ by Section 1.4 and we get an isomorphism $H^{d}\left(X, K_{d+1}\right) \simeq H^{d}\left(X, \mathcal{H}^{d+1}(2)\right)$. Finally, $H^{d}\left(X, \mathcal{H}^{d+1}(2)\right) \simeq H^{d}(X(\mathbb{R}), \mathbb{Z} / 2)$ by [28, (19.5.1)].

In [16, Theorem 5.7], the group $W M S_{d+1}(A)$ is computed when $A$ has a trivial canonical bundle. We have $W M S_{d+1}(A) \simeq H^{d}(X(\mathbb{R}), \mathbb{Z})$ for $d \geq 3$. It follows that the homomorphism $W M S_{d+1}(A) \rightarrow M S_{d+1}(A)$ is in general not injective.

2.2. Prestabilization. Let $A$ be a smooth algebra of dimension $d$ over a field $k$. The natural homomorphism $S L_{d+1}(A) \rightarrow S K_{1}(A)$ is then surjective, and its kernel is $S L_{d+1}(A) \cap E_{d+2}(A)$ by [38]. A matrix in $S L_{d+1}(A) \cap E_{d+2}(A)$ is called 1-stably elementary of size $d+1$. Our goal in this section is to understand this kernel in a cohomological way when the dimension $d$ of $A$ is odd.

Recall from [36, Theorem 5.3 (ii)] that the map $r: S L_{d+1}(A) \rightarrow W M S_{d+1}(A)$ sending a matrix to its first row is a well defined homomorphism. Moreover, when $d$ is odd, we can interpret [36. Theorem 6.1(ii)] by saying that there is an exact sequence

$$
S L_{d+1}(A) \cap E_{d+2}(A) \stackrel{r}{\longrightarrow} W M S_{d+1}(A) \longrightarrow M S_{d+1}(A) \longrightarrow 0 .
$$

It follows that the kernel $K$ of the map $W M S_{d+1}(A) \rightarrow M S_{d+1}(A)$ computes exactly the 1-stably elementary matrices of size $d+1$ whose first row is not completable in an elementary matrix. When $A$ is of odd dimension $d$ over $\mathbb{R}$ and oriented (i.e. $\bigwedge^{d} \Omega_{A / \mathbb{R}} \simeq A$ ), there are a lot of these matrices.

Theorem 2.4. Let $A$ be a smooth affine algebra of odd dimension $d \geq 3$ over $\mathbb{R}$ and let $X=\operatorname{Spec}(A)$. Suppose that $A$ is rational and oriented. Let $\mathcal{C}$ be the set of compact components of $X(\mathbb{R})$. Then $K \simeq \bigoplus_{C \in \mathcal{C}} \mathbb{Z}$.

Proof. First observe that $H^{d}\left(A, I^{j}\right) \simeq \bigoplus_{C \in \mathcal{C}} \mathbb{Z}$ for any $j \geq d$ ([16. Proposition 5.1]). Moreover, the natural homomorphism $H^{d}\left(A, I^{j+1}\right) \rightarrow H^{d}\left(A, I^{j}\right)$ is multiplication by 2 on any compact component of $A(\mathbb{R})$ (and hence is injective). Now the homomorphism $H^{d}\left(A, G^{j}\right) \rightarrow H^{d}\left(A, I^{j}\right)$ is an isomorphism for $j \geq d$ (16. Theorem $5.7])$. We can now conclude that the sequence

$$
H^{d}\left(A, I^{d+2}\right) \longrightarrow H^{d}\left(A, G^{d+1}\right) \longrightarrow H^{d}\left(A, K_{d+1}\right) \longrightarrow 0
$$

is also exact on the left. Hence $K \simeq H^{d}\left(A, I^{d+2}\right) \simeq \bigoplus_{C \in \mathcal{C}} \mathbb{Z}$. 
Example 2.5. If $A=\mathbb{R}\left[x_{1}, \ldots, x_{d+1}\right] /\left(\sum x_{i}^{2}-1\right)$ is an algebraic real sphere of dimension $d \geq 3$, then [16, proof of Corollary 5.12] shows that $W M S_{d+1}(A)$ is generated by $\left[x_{1}, \ldots, x_{d+1}\right]$. If $d=3$, then $\left(x_{1}, \ldots, x_{4}\right)$ is the first row of the symplectic matrix (see [15, Theorem 6.11])

$$
\left(\begin{array}{cccc}
x_{1} & x_{2} & x_{3} & x_{4} \\
-x_{2} & x_{1} & -x_{4} & x_{3} \\
-x_{3} & x_{4} & x_{1} & -x_{2} \\
-x_{4} & -x_{3} & x_{2} & x_{1}
\end{array}\right)
$$

The first row of the square of this matrix

$$
\left(\begin{array}{cccc}
2 x_{1}^{2}-1 & 2 x_{1} x_{2} & 2 x_{1} x_{3} & 2 x_{1} x_{4} \\
-2 x_{1} x_{2} & 2 x_{1}^{2}-1 & -2 x_{1} x_{4} & 2 x_{1} x_{3} \\
-2 x_{1} x_{3} & 2 x_{1} x_{4} & 2 x_{1}^{2}-1 & -2 x_{1} x_{2} \\
-2 x_{1} x_{4} & -2 x_{1} x_{3} & 2 x_{1} x_{2} & 2 x_{1}^{2}-1
\end{array}\right)
$$

represents $2\left[x_{1}, \ldots, x_{4}\right]$ in $W M S_{4}(A)$. Hence its first row is not the first row of an elementary matrix. However,

$$
\left(\begin{array}{ccccc}
2 x_{1}^{2}-1 & 2 x_{1} x_{2} & 2 x_{1} x_{3} & 2 x_{1} x_{4} & 0 \\
-2 x_{1} x_{2} & 1-2 x_{2}^{2} & -2 x_{2} x_{3} & -2 x_{2} x_{4} & 0 \\
-2 x_{1} x_{3} & -2 x_{2} x_{3} & 1-2 x_{3}^{2} & -2 x_{3} x_{4} & 0 \\
-2 x_{1} x_{4} & -2 x_{2} x_{4} & -2 x_{3} x_{4} & 1-2 x_{4}^{2} & 0 \\
0 & 0 & 0 & 0 & 1
\end{array}\right)
$$

is elementary by [40, Lemma 2.2] (with $\mu=\left(\begin{array}{llll}x_{1} & x_{2} & x_{3} & x_{4}\end{array}\right)$ and $\nu=-2\left(\begin{array}{l}x_{1} \\ x_{2} \\ x_{3} \\ x_{4}\end{array}\right)$ ).

In contrast, we have:

Theorem 2.6. Let $k$ be a perfect field such that c.d..2 $(k) \leq 2$ and let $A$ be a smooth affine algebra of dimension $d \geq 3$ over $k$. Then the first row of any 1-stably elementary matrix of size $d+1$ is completable in an elementary matrix.

Proof. If $d$ is odd, then it is a straightforward consequence of Theorem 2.1. If $d$ is even, this is [39, $\S 6$, Theorem 10].

Remark 2.7. If $c . d_{.2}(k) \leq 1$ and $(d+1) ! \in k^{\times}$, then a stronger result is proved in [26.

2.3. About stably free modules. Recall from Section 1.1 that the pointed set $U m_{n}(R) / S L_{n}(R)$ computes the set of isomorphism classes of projective $R$-modules $P$ such that $P \oplus R \simeq R^{n}$. A consequence of Theorem 1.4 is that we get a cohomological description of $U m_{d+1}(A) / S L_{d+1}(A) \cap E_{d+2}(A)$ when $A$ is a smooth algebra of odd dimension $d \geq 3$.

Proposition 2.8. Let $A$ be a smooth algebra of odd dimension $d \geq 3$ over a perfect field $k$ with $\operatorname{char}(k) \neq 2$. Then $U_{d+1}(A) / S L_{d+1}(A) \cap E_{d+2}(A) \simeq H^{d}\left(A, K_{d+1}\right)$.

Proof. This is an easy consequence of [36, Theorem 6.1(ii)]. 
In view of Theorem 2.3 , we get the following corollary.

Corollary 2.9. Let $A$ be a smooth algebra of odd dimension $d \geq 3$ over $\mathbb{R}$. Suppose moreover that $A$ is rational and let $X=\operatorname{Spec}(A)$. Then

$$
U m_{d+1}(A) / S L_{d+1}(A) \cap E_{d+2}(A) \simeq H^{d}(X(\mathbb{R}), \mathbb{Z} / 2) .
$$

Using the homomorphism $r: S L_{d+1}(A) \rightarrow W M S_{d+1}(A)$ sending a matrix to its first row, we get a description of $U m_{d+1}(A) / S L_{d+1}(A)$ over any perfect field.

Theorem 2.10. Let $A$ be a smooth affine algebra of odd dimension $d \geq 3$ over a perfect field $k$. Then $U m_{d+1}(A) / S L_{d+1}(A) \simeq H^{d}\left(A, K_{d+1}\right) / S L_{d+1}(A)$.

Remark 2.11. This result is wrong when the dimension $d$ is even. For example, when $A$ is the real algebraic sphere of dimension 2 , then $U m_{3}(A) / S L_{3}(A)=\mathbb{Z}$ by [16. Corollary 5.12]. On the other hand, $H^{2}\left(X, K_{3}\right)=\mathbb{Z} / 2$.

Remark 2.12. Under the assumptions of the theorem, we have the stabilization isomorphism $S L_{d+1}(A) / S L_{d+1}(A) \cap E_{d+2}(A) \simeq S K_{1}(A)$. The first row homomorphism then yields a homomorphism

$$
r: S K_{1}(A) \rightarrow H^{d}\left(A, K_{d+1}\right)
$$

whose cokernel is precisely $U m_{d+1}(A) / S L_{d+1}(A)$.

\section{The BAss-Kubota theOREM FOR SURFACES}

3.1. Grothendieck-Witt groups. In this section, we briefly recall some basic facts about Grothendieck-Witt groups. The reader is referred to [29, §8] for more information. If $X$ is a scheme over $k$ and $L$ is a line bundle over $X$, then there are abelian groups $G W_{i}^{j}(X, L)$ for any $i \in \mathbb{Z}$ and $j \in \mathbb{Z} / 4$ generalizing the classical Grothendieck-Witt group $G W(X)$. If $X=\operatorname{Spec}(R)$, then $G W_{i}^{0}(R)=K_{i} O(R)$ while $G W_{i}^{2}(R)=K_{i} S p(R)$. The other groups correspond with Karoubi's $U$ and $V$ groups $([30, \S 3.6])$. To compare Quillen $K$-theory and higher Grothendieck-Witt groups, there are two functors

$$
f: G W_{i}^{j}(X, L) \rightarrow K_{i}(X)
$$

and

$$
H: K_{i}(X) \rightarrow G W_{i}^{j}(X, L)
$$

respectively called a forgetful functor and a hyperbolic functor. In this setting, Karoubi periodicity reads as a long exact sequence

$$
\ldots \rightarrow G W_{i}^{j}(X, L) \stackrel{f}{\rightarrow} K_{i}(X) \stackrel{H}{\rightarrow} G W_{i}^{j+1}(X, L) \stackrel{\eta}{\rightarrow} G W_{i-1}^{j}(X, L) \stackrel{f}{\rightarrow} K_{i-1}(X) \rightarrow \ldots
$$

A basic tool to study Grothendieck-Witt groups is the Gersten-Grothendieck-Witt spectral sequence ([12, Theorem 25] or [18, Theorem 1.7]). Namely, for any regular scheme $X$ and any $n \in \mathbb{Z}$ there exists a spectral sequence $E(n)^{p q}$ converging to $G W_{n-p-q}^{n}(X)$ whose terms at page 1 are

$$
E(n)_{1}^{p q}:=\bigoplus_{x_{p} \in X^{(p)}} G W_{n-p-q}^{n-p}\left(k\left(x_{p}\right), \omega_{x_{p}}\right) .
$$

By construction, the hyperbolic and forgetful functors induce a morphism of spectral sequences $H: E^{p q} \rightarrow E(n)^{p q}$ and $f: E(n)^{p q} \rightarrow E^{p q}$, where $E^{p q}$ is the BrownGersten-Quillen spectral sequence in $K$-theory ([32, Theorem 5.20]). 
For our purpose we will need only the following two results, the first one being an easy lemma.

Lemma 3.1. Let $F$ be a field. For $0 \leq n \leq 2$, the image of the homomorphism $\eta: G W_{n}^{n}(F) \rightarrow G W_{n-1}^{n-1}(F)$ is equal to $I^{n}(F)$ and the diagram

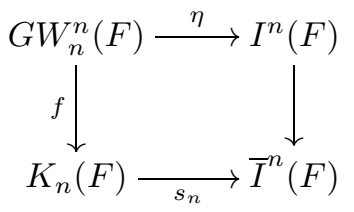

is a fibre product, where $f: G W_{n}^{n}(F) \rightarrow K_{n}(F)$ is the forgetful homomorphism.

Proof. For $n=0$, this is obvious. For $G W_{1}^{1}$, see [3, Corollary 4.5.1.5] and see [34, Corollary 6.4] for $G W_{2}^{2}$.

Proposition 3.2. Let $S$ be a smooth affine surface over an algebraically closed field. Then the forgetful homomorphism $f: K_{1} S p(S) \rightarrow K_{1}(S)$ is split injective.

Proof. We may assume that $S$ is connected. Recall first that $K_{1} S p(S)=G W_{1}^{2}(S)$. We use the Gersten-Grothendieck-Witt spectral sequence $E(2)$ to compute this group. Since $S$ is a surface, the information is concentrated on the lines $q=-1,0,1$. The line $q=1$ is trivial by [12, Lemma 2.2], while the line $q=-1$ is as follows:

$$
G W_{3}^{2}\left(k\left(x_{0}\right)\right) \longrightarrow \bigoplus_{x_{1} \in X^{(1)}} G W_{2}^{1}\left(k\left(x_{1}\right)\right) \longrightarrow \bigoplus_{x_{2} \in X^{(2)}} G W_{1}^{0}\left(k\left(x_{2}\right)\right)
$$

The forgetful functor $f$ induces a commutative diagram

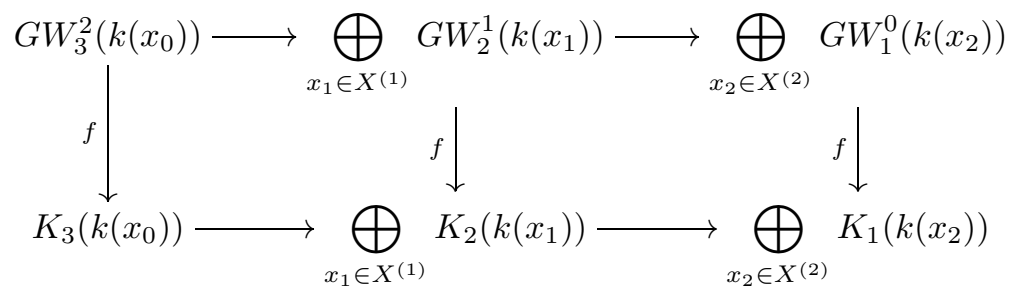

By [12, Lemma 2.4], the forgetful functor $f: G W_{i}^{i-1}(F) \rightarrow K_{i}(F)$ factors through the 2 -torsion and induces a surjective map $f: G W_{i}^{i-1}(F) \rightarrow\{2\} K_{i}(F)$ for any $i=1,2$ and any field $F$. Moreover, if $F$ is algebraically closed, then the map $f: G W_{1}^{0}(F) \rightarrow\{2\} K_{1}(F)$ is an isomorphism. It follows that the cokernel of

$$
\bigoplus_{x_{1} \in X^{(1)}} G W_{2}^{1}\left(k\left(x_{1}\right)\right) \longrightarrow \bigoplus_{x_{2} \in X^{(2)}} G W_{1}^{0}\left(k\left(x_{2}\right)\right)
$$

is isomorphic to $C H^{2}(S) / 2$, which is trivial by [11, Lemma 1.2]. This proves that the spectral sequence induces an isomorphism $K_{1} S p(S) \simeq E(2)_{2}^{1,0}$. Explicitly, $K_{1} S p(S)$ is isomorphic to the homology of the complex

$$
G W_{2}^{2}\left(k\left(x_{0}\right)\right) \longrightarrow \bigoplus_{x_{1} \in X^{(1)}} G W_{1}^{1}\left(k\left(x_{1}\right)\right) \longrightarrow \bigoplus_{x_{2} \in X^{(2)}} G W\left(k\left(x_{2}\right)\right)
$$


Arguing as in the proof of Theorem 2.1, we see that $I^{d+1-j}\left(k\left(x_{j}\right)\right)=0$ for any $x \in X^{(j)}$. Lemma 3.1 then shows that the forgetful functor induces an isomorphism of complexes

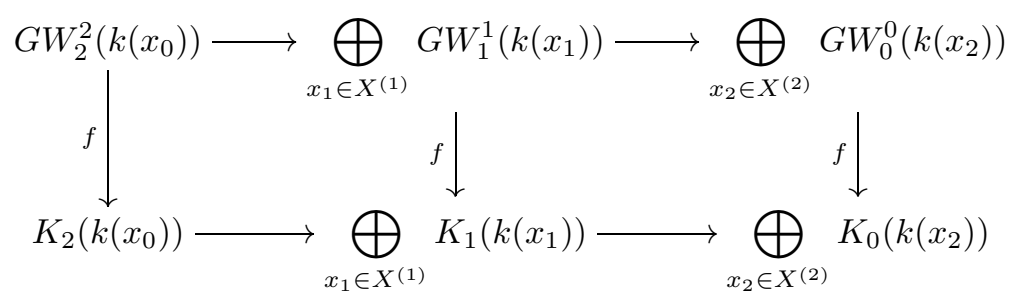

Therefore the forgetful functor $f: K_{1} S p(S) \rightarrow K_{1}(S)$ induces an isomorphism $K_{1} S p(S) \simeq H^{1}\left(S, K_{2}\right)$. Now using the Brown-Gersten-Quillen spectral sequence $E^{p, q}$ converging to the $K$-theory of $S$, we see that $E_{\infty}^{1,-2}=H^{1}\left(S, K_{2}\right)$ and that $f$ in fact induces an isomorphism $K_{1} S p(S) \rightarrow E_{\infty}^{1,-2}$. It follows that $f$ is split injective.

We now have all the tools in hand to discuss the analogue of the Bass-Kubota theorem for surfaces over an algebraically closed field. This is the object of the following subsection.

3.2. Mennicke symbols of length 2 . Let $R$ be a ring. It is well known that in general $E_{2}(R)$ is not a normal subgroup of $S L_{2}(R)$ (see [10 for instance). Our purpose being the description of the group $S K_{1}(R)$ in special cases, we will have to consider slightly modified Mennicke symbols that we call stable Mennicke symbols of rank 2. The definition below is different from the original definition of [5, Definition 2.5] (compare also with [40, (4.3), (4.4)]).

A stable Mennicke symbol of length 2 is a map $m s: U m_{2}(R) \rightarrow G$, where $G$ is a group, such that:

ms1. $m s(1,0)=1$ and $m s(v)=m s(w M)$ if $M \in S L_{2}(R) \cap E(R)$.

ms2. $m s(a, c) \cdot m s(b, c)=m s(a b, c)$ for any unimodular rows $(a, c)$ and $(b, c)$.

We write $M S_{2}(R)$ for the universal stable Mennicke symbol. It follows from [40, §4] that the map

$$
\Phi: U m_{2}(R) \rightarrow S K_{1}(R)
$$

defined by $\Phi(a, b)=\left(\begin{array}{ll}a & b \\ f & e\end{array}\right)$, where $e, f$ are any elements in $R$ such that $a e-b f=1$, is a stable Mennicke symbol of length 2 .

A symplectic stable Mennicke symbol is a map $m s p: U m_{2}(R) \rightarrow G$, where $G$ is a group, such that:

$\operatorname{msp} 1 . m s p(1,0)=1$ and $m s p(v)=m s p(w M)$ if $M \in S L_{2}(R) \cap E S p(R)$. msp 2. $m s p(a, b) \cdot m s p\left(a, c^{2}\right)=m s p\left(a, b c^{2}\right)$ for any $(a, b),\left(a, c^{2}\right) \in U m_{2}(R)$.

We denote by $M S p_{2}(R)$ the universal symplectic stable Mennicke symbol. By [40, §4] again, the map $\Phi$ above factorizes through $K_{1} S p(R)$, yielding a map

$$
\Psi: U m_{2}(R) \rightarrow K_{1} S p(R)
$$

which is a symplectic stable Mennicke symbol. If $R$ is of dimension 2 , then $U m_{4}(R)=e_{1} E_{4}(R)=e_{1} E S p_{4}(R)$ by [40, $\S 4$ ]. It follows that $\Psi$ induces a surjective homomorphism $M S p_{2}(R) \rightarrow K_{1} S p(R)$. 
Lemma 3.3. Let $S$ be a smooth affine surface over an algebraically closed field of characteristic different from 2,3. Then

$$
S L_{2}(S) \cap E(S)=S L_{2}(S) \cap E_{3}(S)=S L_{2}(S) \cap E S p_{4}(S)=S L_{2}(S) \cap E S p(S) .
$$

Proof. The diagram

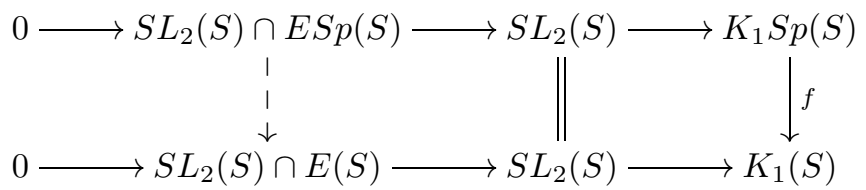

and Proposition 3.2 show that $S L_{2}(S) \cap E S p(S)=S L_{2}(S) \cap E(S)$. Now

$$
S L_{2}(S) \cap E S p(S)=S L_{2}(S) \cap E S p_{4}(S)
$$

by [6. Theorem 2] and $S L_{2}(S) \cap E(S)=S L_{2}(S) \cap E_{3}(S)$ by [26, $\left.\S 3\right]$.

It follows from this lemma that $M S_{2}(S)$ is a symplectic stable Mennicke symbol. Therefore:

Corollary 3.4. Let $S$ be a smooth affine surface over an algebraically closed field of characteristic different from 2,3. Then there is a unique homomorphism

$$
\alpha: M S p_{2}(S) \rightarrow M S_{2}(S)
$$

such that the diagram

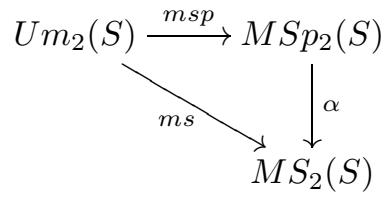

commutes.

In order to prove the analogue of Bass-Kubota theorem for surfaces over the algebraic closure of a finite field, we will need a few preparatory results:

Lemma 3.5 (Mennicke-Newman lemma). Let $S$ be an affine surface over the algebraic closure $k$ of a finite field. Given unimodular rows $(a, b)$ and $(c, d)$, there exist elementary matrices $M_{1}, M_{2} \in E_{2}(S)$ such that if $\left(a^{\prime}, b^{\prime}\right):=(a, b) M_{1}$ and $\left(c^{\prime}, d^{\prime}\right):=(c, d) M_{2}$, we have $\left(a^{\prime}, c^{\prime}\right)$ unimodular.

Proof. Consider the unimodular row $(a, c, b d) \in U m_{3}(S)$. By Vaserstein's theorem [40, Corollary 17.3], there exists $e \in S$ such that $(a+e b d, c+e b d) \in U m_{2}(S)$. We set $M_{1}=\left(\begin{array}{cc}1 & 0 \\ e d & 1\end{array}\right)$ and $M_{2}=\left(\begin{array}{cc}1 & 0 \\ e b & 1\end{array}\right)$.

Lemma 3.6. Let $R$ be a ring and $(a, b),(c, d) \in U m_{2}(R)$ such that $(a, c) \in U m_{2}(R)$. Then

$$
m s(a, b) \cdot m s(c, d)=m s\left((a, b) \cdot\left(\begin{array}{ll}
c & d \\
e & f
\end{array}\right)\right)
$$

in $M S_{2}(R)$ for any $e, f \in R$ such that $c f-d e=1$. 
Proof. First observe that there exists $e, f \in R$ such that $c f-d e=1$ and $(a, f)$ is unimodular. Indeed, since $(c, a d) \in U m_{2}(R)$ there exists $\lambda, \mu \in R$ such that $\lambda c+\mu a d=1$. It suffices to choose $f=\lambda$ and $e=-\mu a$.

Given such $e, f \in R$, let $\alpha:=m s(a c+b e, a d+b f) \cdot m s(-f, d) \cdot m s(f, a)$. Since $\left(\begin{array}{cc}-1 & 0 \\ 0 & -1\end{array}\right) \in E_{2}(R)$, we have

$$
\begin{aligned}
\alpha & =m s(a c+b e, a d+b f) \cdot m s(-f, d) \cdot m s(-f,-a) \\
& =m s(a c+b e, a d+b f) \cdot m s(-f,-a d) \\
& =m s(a c+b e, a d+b f) \cdot m s(f, a d) \\
& =m s(a c+b e, a d+b f) \cdot m s(f, a d+b f) \\
& =m s(f(a c+b e), a d+b f) \\
& =m s(f(a c+b e)-e(a d+b f), a d+b f) \\
& =m s(a, a d+b f) \\
& =m s(a, b f) .
\end{aligned}
$$

As $\left(\begin{array}{cc}0 & -1 \\ 1 & 0\end{array}\right) \in E_{2}(R)$, we get

$$
\begin{aligned}
m s(a, b) \cdot m s(c, d) \cdot m s(-f, d) \cdot m s(f, a) & =m s(a, b) \cdot m s(f, a) \\
& =m s(a, b) \cdot m s(a,-f) \\
& =m s(a,-b f) .
\end{aligned}
$$

It suffices therefore to prove that $m s(a, b f)=m s(a,-b f)$ to conclude. Using again that $\left(\begin{array}{cc}-1 & 0 \\ 0 & -1\end{array}\right)$ is in $E_{2}(R)$, we obtain

$$
\begin{aligned}
m s(a, b f) & =m s(a, b) \cdot m s(a, f)=m s(-a,-b) \cdot m s(-a,-f) \\
& =m s(-a, b f)=m s(a,-b f) .
\end{aligned}
$$

Corollary 3.7. Let $S$ be a smooth affine surface over the algebraic closure of a finite field of characteristic different from 2 and 3. Then the stable Mennicke symbol $\Phi: U m_{2}(S) \rightarrow S K_{1}(S)$ is universal and the map ms:Um $U m_{2}(S) \rightarrow M S_{2}(S)$ induces a bijection

$$
U m_{2}(S) / S L_{2}(S) \cap E_{3}(S) \simeq M S_{2}(S) .
$$

Proof. It follows from Lemmas 3.5 and 3.6 that the map ms: $U m_{2}(S) \rightarrow M S_{2}(S)$ is surjective. By Lemma 3.3 , we obtain a surjective map

$$
m s: U m_{2}(S) / S L_{2}(S) \cap E_{3}(S) \rightarrow M S_{2}(S) .
$$

Now $\Phi: U m_{2}(S) \rightarrow S K_{1}(S)$ induces an injective map

$$
\Phi: U m_{2}(S) / S L_{2}(S) \cap E_{3}(S) \rightarrow S K_{1}(S)
$$

which is also surjective by [40, Corollary 7.3]. Finally, $\Phi$ is a stable Mennicke symbol, and therefore there exists a homomorphism $f: M S_{2}(S) \rightarrow S K_{1}(S)$ such 
that the diagram

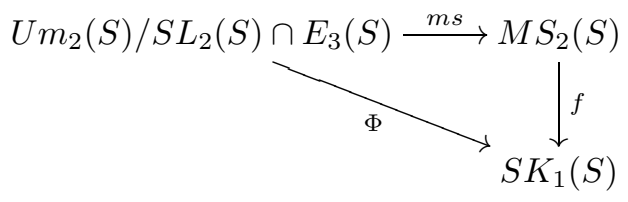

commutes. It follows that $m s$ is also injective and that $f$ is an isomorphism.

Remark 3.8. In a previous version of this paper, we asserted that the map

$$
U m_{2}(S) / S L_{2}(S) \cap E_{3}(S) \rightarrow M S_{2}(S)
$$

was a bijection for any algebraically closed field of characteristic different from 2 and 3. However, as pointed out by the referee, the Mennicke-Newman lemma doesn't hold in this generality and it is not clear that the map is surjective.

To conclude, we bring the universal symplectic stable Mennicke symbol in the picture.

Theorem 3.9. Let $S$ be a smooth affine surface over the algebraic closure of a finite field of characteristic different from 2 and 3 . Then the maps in the diagram

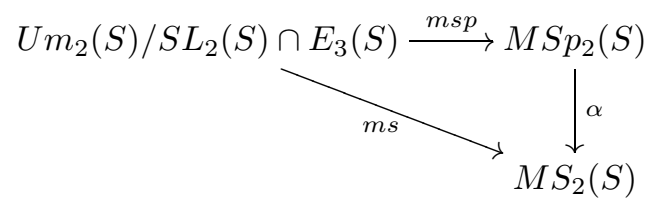

are bijections.

Proof. In view of Corollary [3.7, it suffices to show that $m s p$ is surjective. Consider the map

$$
\theta: U m_{2}(S) \rightarrow M S p_{2}(S)
$$

defined by $\theta(a, b)=m s p\left(a^{2}, b\right)$. It is not hard to check that $\theta$ satisfies rules ms1. and ms2. and therefore it induces a homomorphism $f: M S_{2}(S) \rightarrow M S p_{2}(S)$ such that $f(m s(a, b))=m s p\left(a, b^{2}\right)$. Using Corollary 3.7, we see that it suffices to prove that $f$ is surjective to deduce the surjectivity of $m s p$. To do this, it suffices to show that given $(a, b) \in U m_{2}(S)$ there exists $(c, d) \in U m_{2}(S)$ such that $m s p(a, b)=m s p\left(c, d^{2}\right)$.

Now $U m_{2}(S) / S L_{2}(S) \cap E S p_{4}(S) \simeq M S_{2}(S)=S K_{1}(S)$ and it suffices to prove that $S K_{1}(S)$ is 2-divisible to conclude. We use the Brown-Gersten-Quillen spectral sequence $E^{p, q}$ to compute $S K_{1}(S)$. By definition, $E_{2}^{2,-3}=H^{2}\left(S, K_{3}\right)$. Lemma 1.3 yields a homomorphism $M S_{3}(S) \rightarrow H^{2}\left(S, K_{3}\right)$ which is surjective by [16, proof of Proposition 4.3]. Since $U m_{3}(S) / E_{3}(S)=0$ by [40, Corollary 17.3], we get $S K_{1}(S)=E_{\infty}^{1,-2}=H^{1}\left(S, K_{2}\right)$. The proof of Theorem 2.2 applies to prove that this group is divisible (not uniquely though).

\section{ACKNOWLEDGMENTS}

It is a pleasure to thank Ravi Rao for useful conversations, and also for the motivation to write the results of this paper. Part of this work was achieved during a beautiful tour at Penn State University and the University of Chicago. Special thanks are due to P. Baum, L. Vaserstein, M. P. Murthy and M. V. Nori for making this possible. The author also wishes to express gratitude to the referee for pointing 
out several mistakes in a previous version of the paper. Finally, a special thanks to W. van der Kallen for a discussion on the differences between Newman and Newton.

\section{REFERENCES}

[1] Jón Kristinn Arason and Albrecht Pfister, Beweis des Krullschen Durchschnittsatzes für den Wittring (German), Invent. Math. 12 (1971), 173-176. MR0294251 (45 \#3320)

[2] L. Barbieri-Viale, C. Pedrini, and C. Weibel, Roitman's theorem for singular complex projective surfaces, Duke Math. J. 84 (1996), no. 1, 155-190, DOI 10.1215/S0012-7094-96-08405-7. MR:1394751 (97c:14004)

[3] Jean Barge and Jean Lannes, Suites de Sturm, indice de Maslov et périodicité de Bott (French), Progress in Mathematics, vol. 267, Birkhäuser Verlag, Basel, 2008. MR2431916 (2010d:19004)

[4] Jean Barge and Fabien Morel, Groupe de Chow des cycles orientés et classe d'Euler des fibrés vectoriels (French, with English and French summaries), C. R. Acad. Sci. Paris Sér. I Math. 330 (2000), no. 4, 287-290, DOI 10.1016/S0764-4442(00)00158-0. MR1753295(2000m:14004)

[5] H. Bass, J. Milnor, and J.-P. Serre, Solution of the congruence subgroup problem for $\mathrm{SL}_{n}(n \geq 3)$ and $\mathrm{Sp}_{2 n}(n \geq 2)$, Inst. Hautes Études Sci. Publ. Math. 33 (1967), 59-137. MR0244257 (39 \#5574)

[6] Rabeya Basu and Ravi A. Rao, Injective stability for $K_{1}$ of classical modules, J. Algebra 323 (2010), no. 4, 867-877, DOI 10.1016/j.jalgebra.2009.12.012. MR.2578583 (2011e:19006)

[7] S. M. Bhatwadekar and Raja Sridharan, On Euler classes and stably free projective modules, Algebra, arithmetic and geometry, Part I, II (Mumbai, 2000), Tata Inst. Fund. Res. Stud. Math., vol. 16, Tata Inst. Fund. Res., Bombay, 2002, pp. 139-158. MR1940666 (2003i:13012)

[8] S. Bloch, Torsion algebraic cycles, $K_{2}$, and Brauer groups of function fields, The Brauer group (Sem., Les Plans-sur-Bex, 1980), Lecture Notes in Math., vol. 844, Springer, Berlin, 1981, pp. 75-102. MR611866 (82k:14018)

[9] Spencer Bloch and Arthur Ogus, Gersten's conjecture and the homology of schemes, Ann. Sci. École Norm. Sup. (4) 7 (1974), 181-201 (1975). MR0412191 (54 \#318)

[10] P. M. Cohn, On the structure of the $\mathrm{GL}_{2}$ of a ring, Inst. Hautes Études Sci. Publ. Math. 30 (1966), 5-53. MR0207856 (34 \#7670)

[11] J.-L. Colliot-Thélène and C. Scheiderer, Zero-cycles and cohomology on real algebraic varieties (English, with English and French summaries), Topology 35 (1996), no. 2, 533-559, DOI 10.1016/0040-9383(95)00015-1. MR1380515 (97a:14009)

[12] J. Fasel and V. Srinivas, Chow-Witt groups and Grothendieck-Witt groups of regular schemes, Adv. Math. 221 (2009), no. 1, 302-329, DOI 10.1016/j.aim.2008.12.005. MR2509328 (2010g:13011)

[13] Jean Fasel, The Chow-Witt ring, Doc. Math. 12 (2007), 275-312. MR2350291 (2008j:14004)

[14] Jean Fasel, Groupes de Chow-Witt, Mém. Soc. Math. Fr. (N.S.) 113 (2008), viii+197. $\operatorname{MR} 2542148$

[15] J. Fasel, Projective modules over the real algebraic sphere of dimension 3, J. Algebra 325 (2011), 18-33, DOI 10.1016/j.jalgebra.2010.09.039. MR2745527 (2011m:13013)

[16] Jean Fasel, Some remarks on orbit sets of unimodular rows, Comment. Math. Helv. 86 (2011), no. 1, 13-39, DOI 10.4171/CMH/216. MR2745274 (2012d:13017)

[17] Jean Fasel, Ravi A. Rao, and Richard G. Swan, On stably free modules over affine algebras, Publ. Math. Inst. Hautes Études Sci. 116 (2012), no. 1, 223-243. MR3090257

[18] Jens Hornbostel, Oriented Chow groups, Hermitian K-theory and the Gersten conjecture, Manuscripta Math. 125 (2008), no. 3, 273-284, DOI 10.1007/s00229-007-0148-5. MR2373061 (2008j:19011)

[19] Kazuya Kato, Milnor K-theory and the Chow group of zero cycles, theory, Part I, II (Boulder, Colo., 1983), Contemp. Math., vol. 55, Amer. Math. Soc., Providence, RI, 1986, pp. 241-253, DOI 10.1090/conm/055.1/862638. MR862638(88c:14012)

[20] Tomio Kubota, Ein arithmetischer Satz über eine Matrizengruppe (German), J. Reine Angew. Math. 222 (1966), 55-57. MR.0188194(32 \#5633)

[21] A. S. Merkur'ev and A. A. Suslin, K-cohomology of Severi-Brauer varieties and the norm residue homomorphism (Russian), Izv. Akad. Nauk SSSR Ser. Mat. 46 (1982), no. 5, 10111046, 1135-1136. MR675529(84i:12007) 
[22] James S. Milne, Étale cohomology, Princeton Mathematical Series, vol. 33, Princeton University Press, Princeton, N.J., 1980. MR559531 (81j:14002)

[23] John Milnor, Algebraic K-theory and quadratic forms, Invent. Math. 9 (1969/1970), 318-344. MR0260844 (41 \#5465)

[24] Fabien Morel, Sur les puissances de l'idéal fondamental de l'anneau de Witt (French, with English and French summaries), Comment. Math. Helv. 79 (2004), no. 4, 689-703, DOI 10.1007/s00014-004-0815-z. MR2099118 (2005f:19004)

[25] D. Orlov, A. Vishik, and V. Voevodsky, An exact sequence for $K_{*}^{M} / 2$ with applications to quadratic forms, Ann. of Math. (2) 165 (2007), no. 1, 1-13, DOI 10.4007/annals.2007.165.1. MR2276765(2008c:19001)

[26] Ravi A. Rao and Wilberd van der Kallen, Improved stability for $S K_{1}$ and $W M S_{d}$ of a nonsingular affine algebra, Astérisque 226 (1994), 11, 411-420. K-theory (Strasbourg, 1992). MR1317126 (96e:19001)

[27] Markus Rost, Chow groups with coefficients, Doc. Math. 1 (1996), No. 16, 319-393 (electronic). MR:1418952 (98a:14006)

[28] Claus Scheiderer, Real and étale cohomology, Lecture Notes in Mathematics, vol. 1588, Springer-Verlag, Berlin, 1994. MR 1321819 (96c:14018)

[29] Marco Schlichting, The Mayer-Vietoris principle for Grothendieck-Witt groups of schemes, Invent. Math. 179 (2010), no. 2, 349-433, DOI 10.1007/s00222-009-0219-1. MR2570120 (2011m:19005)

[30] Marco Schlichting, Hermitian K-theory, derived equivalences and Karoubi's fundamental theorem, arXiv:1209.0848, 2012.

[31] Jean-Pierre Serre, Cohomologie Galoisienne, Lecture Notes in Mathematics, Vol. 5, SpringerVerlag, Berlin, 1973. Cours au Collège de France, Paris, 1962-1963; Avec des textes inédits de J. Tate et de Jean-Louis Verdier; Quatrième édition. MR.0404227 (53 \#8030)

[32] V. Srinivas, Algebraic K-theory, 2nd ed., Progress in Mathematics, vol. 90, Birkhäuser Boston Inc., Boston, MA, 1996. MR1382659 (97c:19001)

[33] A. A. Suslin, Mennicke symbols and their applications in the K-theory of fields, Algebraic $K$-theory, Part I (Oberwolfach, 1980), Lecture Notes in Math., vol. 966, Springer, Berlin, 1982, pp. 334-356. MR689382(84f:18023)

[34] A. A. Suslin, Torsion in $K_{2}$ of fields, $K$-Theory 1 (1987), no. 1, 5-29, DOI 10.1007/BF00533985. MR899915 (89a:11123)

[35] A. A. Suslin, The cancellation problem for projective modules, and related questions (Russian), Proceedings of the International Congress of Mathematicians (Helsinki, 1978), Acad. Sci. Fennica, Helsinki, 1980, pp. 323-330. MR.562623 (83e:13011)

[36] Wilberd van der Kallen, A module structure on certain orbit sets of unimodular rows, J. Pure Appl. Algebra 57 (1989), no. 3, 281-316, DOI 10.1016/0022-4049(89)90035-2. MR987316 (90b:18013)

[37] Wilberd van der Kallen, From Mennicke symbols to Euler class groups, Algebra, arithmetic and geometry, Part I, II (Mumbai, 2000), Tata Inst. Fund. Res. Stud. Math., vol. 16, Tata Inst. Fund. Res., Bombay, 2002, pp. 341-354. MR1940672 (2003h:19006)

[38] L. N. Vaseršteĭn, On the stabilization of the general linear group over a ring, Math. USSR-Sb. 8 (1969), 383-400. MR.0267009 (42 \#1911)

[39] Leonid N. Vaserstein, Computation of $K_{1}$ via Mennicke symbols, Comm. Algebra 15 (1987), no. 3, 611-656, DOI 10.1080/00927878708823434. MR882801(88m:18019)

[40] L. N. Vaseršteĭn and A. A. Suslin, Serre's problem on projective modules over polynomial rings, and algebraic K-theory (Russian), Izv. Akad. Nauk SSSR Ser. Mat. 40 (1976), no. 5, 993-1054, 1199. MR0447245 (56 \#5560)

[41] Vladimir Voevodsky, Motivic cohomology with Z/2-coefficients, Publ. Math. Inst. Hautes Études Sci. 98 (2003), 59-104, DOI 10.1007/s10240-003-0010-6. MR2031199 (2005b:14038b)

[42] C. Weibel, The norm residue isomorphism theorem, J. Topol. 2 (2009), no. 2, 346-372, DOI 10.1112/jtopol/jtp013. MR2529300(2011a:14039)

Fakultät Mathematik, Universität Duisburg-Essen, Campus Essen, Thea-LeymannStrasse 9, D-45127 Essen, Germany

E-mail address: jean.fasel@gmail.com 